Volume 25 (2019) 64-76

DOI: $10.24330 /$ ieja.504114

\title{
FRATTINI PROPERTIES AND NILPOTENCY IN LEIBNIZ ALGEBRAS
}

\author{
Allison McAlister, Kristen Stagg Rovira and Ernie Stitzinger \\ Received: 23 February 2018; Revised: 25 April 2018; Accepted: 7 May 2018 \\ Communicated by James Zhang \\ Abstract. Ideals that share properties with the Frattini ideal of a Leibniz \\ algebra are studied. Similar investigations have been considered in group the- \\ ory. The results will hold for Lie algebras as well. Many of the results involve \\ nilpotency of these algebras.
}

Mathematics Subject Classification (2010): 17A32

Keywords: Frattini ideal, Cartan subalgebra, primitive ideal, non-generator

\section{Introduction}

Frattini theory for algebras goes back at least 50 years. A general theory can be found in [14] and there are many works on special classes of algebras, especially Lie algebras. Leibniz algebras, as a generalization of Lie algebras, is a natural class to investigate and [2] - [9] contain results on Frattini subalgebras and ideals. Frattini theory for groups goes back to the nineteenth century and there have been many results that are similar in groups and Lie algebras. Subgroups that have Frattini like properties have been considered in [7] and related special types of subgroups have been studied in [11]. It is the purpose of this paper to view the Leibniz algebra analogues to some of these theories. Many of these results are new for Lie algebras as well. We consider only finite dimensional Leibniz algebras over a field $F$. The intersection of all maximal subalgebras of $A$ is called the Frattini subalgebra of $A$ and is denoted by $F(A)$. Even in the solvable case, it need not be an ideal in A [5] and the unique maximal one among the ideals of $A$ contained in $F(A)$ is called the Frattini ideal of $A$ and denoted by $\Phi(\mathrm{A})$. References for Leibniz algebras include [1], [2], [10], and [12].

\section{Generalized Frattini ideals}

In [7], a proper subgroup, $H$, of a finite group $G$ is called generalized Frattini if whenever $G=H N_{G}(P)$ for any Sylow subgroup $P$ of any normal subgroup $K$ of $G$, then $G=N_{G}(P)$. To consider such a property in Leibniz algebras, we replace Sylow 
subgroups with Cartan subalgebras. Unlike group theory, Frattini subalgebras do not have to be invariant and we will use the Frattini ideal as our model to be formalized. To guarantee existence of Cartan subalgeras, we assume the algebras are over an infinite field, [2, Theorem 6.5]. In this context, an ideal $H$ of $L$ is generalized Frattini in $A$ if whenever $A=H+N_{A}(C), C$ a Cartan subalgebra of an ideal $K$ in $A$, it follows that $A=N_{A}(C)$. We will show $H$ is a generalized Frattini ideal of $A$ if and only if whenever $D$ and $B$ are ideals of $A$ and $D$ is contained in $B \cap H$, then $B / D$ being nilpotent implies that $B$ is nilpotent, which is a property possessed by Frattini ideals. We will find examples of this concept and conditions that guarantee that an ideal is generalized Frattini.

Proposition 2.1. Let $H$ be a generalized Frattini ideal in A. Then the following are true.

(1) $H$ is nilpotent.

(2) Any ideal of $A$ that is contained in $H$ is also a generalized Frattini ideal in A.

(3) $H+\Phi(A)$ is a generalized Frattini ideal in $A$.

(4) $H+Z(A)$ is a generalized Frattini ideal in $A$ whenever $H+Z(A)$ is a proper subalgebra of $A$.

Proof. (1) Let $C$ be a Cartan subalgebra of $H$. Then $A=H+N_{A}(C)$ by Theorem 6.6 in [2]. $H$ is generalized Frattini in $A$, hence $A=N_{A}(C)$. Therefore, $H=$ $N_{H}(C)$. Since $C$ is a Cartan subalgebra of $H, N_{H}(C)=C$. Thus $H=C$ and $H$ is nilpotent.

(2) Let $N$ be an ideal of $A$ such that $N \subseteq H$. Let $K$ be an ideal of $A$ and let $C$ be a Cartan subalgebra of $K$ such that $A=N+N_{A}(C)$. Then $A=H+N_{A}(C)$ and, hence, $A=N_{A}(C)$ since $H$ is generalized Frattini in $A$. Thus by definition, $N$ is also generalized Frattini in $A$.

(3) Let $K$ be an ideal in $A$ with Cartan subalgebra $C$ such that $A=H+\Phi(A)+$ $N_{A}(C)$. Suppose that $M$ is a maximal subalgebra of $A$ such that $H+N_{A}(C) \subseteq M$. Then $H+\Phi(A)+N_{A}(C) \subseteq M$, a contradiction. Hence $A=H+N_{A}(C)$. Therefore $A=N_{A}(C)$ since $H$ is generalized Frattini in $A$. Therefore $H+\Phi(A)$ is generalized Frattini in $A$.

(4) Suppose that $K$ is an ideal in $A$ with Cartan subalgebra $C$ such that $A=$ $H+Z(A)+N_{A}(C)$. Then $A=H+N_{A}(C)$ and $A=N_{A}(C)$ since $H$ is generalized Frattini in $A$. Therefore $H+Z(A)$ is also generalized Frattini in $A$. 
Corollary 2.2. In a non-abelian Leibniz algebra, $A$, both $Z(A)$ and $\Phi(A)$ are generalized Frattini in A.

Lemma 2.3. Any proper ideal, $H$, of a nilpotent Leibniz algebra $A$ is generalized Frattini in $A$.

Proof. Let $K$ be an ideal in $A$ with Cartan subalgebra $C$ such that $H+N_{A}(C)=A$. Then $C=K$ and $N_{A}(C)=A$.

The next result shows that an important property of the Frattini ideal is shared with any generalized Frattini ideal.

Theorem 2.4. Let $H$ be generalized Frattini in $A$. If $K$ is an ideal in $A$ that contains $H$ and $K / H$ is nilpotent, then $K$ is nilpotent.

Proof. Let $K$ be as in the statement of the theorem and let $C$ be a Cartan subalgebra of $K$. Then $(C+H) / H$ is a Cartan subalgebra of $K / H$ by Corollary 6.3 of [2]. Since $K / H$ is nilpotent, $K / H=(C+H) / H$ and $K=C+H$. Furthermore $A=K+N_{A}(C)$ by Theorem 6.6 of [2]. Then $A=K+N_{A}(C)=H+C+N_{A}(C)=$ $H+N_{A}(C)=N_{A}(C)$ since $H$ is generalized Frattini. Hence $K=N_{K}(C)=C$ and $K$ is nilpotent.

Corollary 2.5. Suppose that $A$ is not $0 . A$ is nilpotent if and only if $A^{2}$ is generalized Frattini.

Proof. If $A^{2}$ is generalized Frattini, then the result follows from Theorem 2.4. If $A$ is nilpotent, then the result follows from Lemma 2.3.

Corollary 2.6. Let $H$ be generalized Frattini in $A$. If $K$ is an ideal in $A$ such that $K^{\omega} \subseteq H$. Then $K$ is nilpotent.

Proof. Let $\sigma$ be the natural mapping from $K / K^{\omega}$ onto $K+H / H . K+H / H$ is nilpotent and then $K+H$ is nilpotent by Theorem 2.4 .

Theorem 2.7. Let $H$ be an ideal in a Leibniz algebra $A . H$ is generalized Frattini in $A$ if and only if for each ideal $J$ of $A$ that contains $H$, whenever $J / H$ is nilpotent, then $J$ is nilpotent.

Proof. If $H$ is generalized Frattini, then the result is Theorem 2.4. Conversely, suppose that the condition on ideals $J$ holds. Let $K$ be an ideal of $A, C$ a Cartan subalgebra of $K$ with $A=H+N_{A}(C)$. Then $(C+H) / H$ is an ideal in $A / H$, hence also in $(K+H) / H$. As $H$ and $K$ are ideals in $L$ and $C$ is a Cartan subalgebra of $K$, 
by Corollary 6.3 of [2], $C+(K \cap H) /(K \cap H)$ is Cartan in $K /(K \cap H)$. By the first isomorphism theorem, $C+H / H$ is Cartan in $K+H / H$. Since $(C+H) / H$ is Cartan in $(K+H) / H,(C+H) / H=(K+H) / H$. Therefore $(K+H) / H$ is nilpotent and $K+H$ is nilpotent by hypothesis. Therefore, $K=C$. Hence $N_{A}(C)=N_{A}(K)=A$ and $H$ is generalized Frattini in $A$.

Example 2.8. Let $A$ be a Leibniz algebra with basis $x, y, z$ and multiplications $x z=x=-z x$ and $z y=y=-y z$ and $x y=y x=0$. Let $H=(x)$ and $K=(y)$. $H$ and $K$ are generalized Frattini in $A$ but $H+K$ is not. Thus the sum of two generalized Frattini ideals need not be generalized Frattini. Note that this example is Lie so the result stands in Lie algebras as well.

Theorem 2.9. Let $H$ be a generalized Frattini ideal in $A$ and let $K$ be an ideal of $A$ that contains $H$. Then $K / H$ is generalized Frattini in $A / H$ if and only if $K$ is generalized Frattini in A.

Proof. Suppose that $K$ is generalized Frattini in $A$. Let $J / H$ be an ideal in $A / H$ such $J / H$ contains $K / H$ and $(J / H) /(K / H)$ is nilpotent. Then $J / K$ is nilpotent. Since $K$ is generalized Frattini in $A, J$ is nilpotent by Theorem 2.7. Hence $J / H$ is nilpotent and $K / H$ is generalized Frattini in $A / H$ by Theorem 2.7.

Conversely, suppose that $K / H$ is generalized Frattini in $A / H$. Let $J$ be an ideal in $A$ which contains $K$ and $J / K$ is nilpotent. Then $(J / H) /(K / H)$ is nilpotent. Hence $J / H$ is nilpotent since $K / H$ is generalized Frattini and, then, $J$ is nilpotent since $H$ is generalized Frattini. Therefore $K$ is generalized Frattini in $A$ by Theorem 2.7 .

Proposition 2.10. If $N$ il $(A)$ is generalized Frattini in $A$, then every solvable ideal of $A$ is nilpotent and is generalized Frattini in $A$.

Proof. Suppose that $\operatorname{Nil}(A)$ is generalized Frattini in $A$, let $H$ be a solvable ideal in $A$ and $\mathrm{k}$ be the smallest positive integer such that $H^{(k+1)}=0$. Then $H^{(k)}$ is abelian and $H^{(k)} \subseteq N i l(A)$. Then $H^{(k)}$ is generalized Frattini in $A$ by Proposition 2.1. Consider $A / H^{k}$. The nilradical of this quotient, $N=N i l\left(A / H^{k}\right)$, is isomorphic to $\operatorname{Nil}(A) / H^{k}$ using Theorem 2.7 and $N$ is generalized Frattini by Theorem 2.9. By induction, $H / H^{k}$ is contained in $N$ which gives $H \subseteq N i l(A)$. Thus $H$ is nilpotent and it is contained in a generalized Frattini ideal, $\operatorname{Nil}(A)$. Hence $H$ is generalized Frattini in $A$.

Corollary 2.11. If $N i l(A)$ is generalized Frattini in $A$, then $A$ is not solvable. 
Proof. $A$ is not nilpotent since a generalized Frattini ideal is a proper ideal. If $A$ is solvable, then it is nilpotent by Proposition 2.10, a contradiction.

Example 2.12. Continuing Example 2.8, $N i l(A)=H+K$ is again seen to be not generalized Frattini since $A$ is solvable.

Proposition 2.13. If $H$ is generalized Frattini in $A$, then $N i l(A / H)=N i l(A) / H$.

Proof. Since $H$ is nilpotent, $H \subseteq \operatorname{Nil}(A)$. $N i l(A) / H$ is clearly contained in $\operatorname{Nil}(A / H)$. Suppose that $B$ is an ideal of $A$ such that $B / H=N i l(A / H)$. Then $B$ is nilpotent by Theorem 2.4. Hence $B$ is contained in $N i l(A)$ and $B / H$ is contained in $\operatorname{Nil}(A) / H$.

Corollary 2.14. Let $A$ be a non-nilpotent Leibniz algebra. Then Nil( $A)$ is generalized Frattini in $A$ if and only if $\operatorname{Nil}(A)=\operatorname{Rad}(A)$, where $\operatorname{Rad}(A)$ is the maximal solvable ideal of $A$.

Proof. Suppose that $\operatorname{Nil}(A)=\operatorname{Rad}(A)$. Let $N$ be an ideal in $A$ containing $\operatorname{Nil}(A)$ such that $N / \operatorname{Nil}(A)$ is nilpotent. Then $N$ is solvable and $N i l(A) \subseteq N \subseteq \operatorname{Rad}(A)=$ $\operatorname{Nil}(A)$. Hence $N$ is nilpotent and $\operatorname{Nil}(A)$ is generalized Frattini in $A$ by Theorem 2.7. Conversely, suppose that $\operatorname{Nil}(A)$ is generalized Frattini in $A$. Then $\operatorname{Rad}(A)$ is nilpotent by Proposition 2.10 and $\operatorname{Nil}(A)=\operatorname{Rad}(A)$.

Example 2.15. Let $A=\operatorname{gl}(n, F)$. Then $\operatorname{Nil}(A)=\operatorname{Rad}(A)=Z(A)$ is generalized Frattini in $A$.

In [2] and [3] Barnes extends his theory of Engel subalgebras from Lie to Leibniz algebras. For $a \in A$, set $E_{A}(a)$ be the Fitting null component of left multiplication by $a$ on $A$. This space is a subalgebra called the Engel subalgebra for $a$. He notes that although $a$ may not be in $E_{A}(a)$, there is a $b$ in $E_{A}(a)$ such that $E_{A}(a)=E_{A}(b)$. Hence when working with these subalgebras, we usually can assume that $a$ is in $E_{A}(a)$. For a subalgebra $U$ of $A$, if the Engel subalgebra for $u$ in $U$ both contains $U$ and is minimal in the set of Engel subalgebras for all elements in $U$, then the Engel subalgebras for all elements in $U$ contain $U$. He then shows $C$ is a Cartan subalgebra of $A$ if and only if $C$ is minimal in the set of Engel subalgebras of $A$.

Theorem 2.16. Let $H$ be an ideal in $A$. Then $H$ is generalized Frattini in $A$ if and only if for each ideal $K$ of $A$ and each Cartan subalgebra $C$ of $K, A=E_{A}(c)$ whenever $A=H+E_{A}(c)$, for all $c \in C$.

Proof. Let $H$ be generalized Frattini in $A$. Let $K, C$ be as in the theorem such that for each $c \in C, A=H+E_{A}(c)$. 
As $H$ and $K$ are ideals in $L$ and $C$ is a Cartan subalgebra of $K$, by Corollary 6.3 of [2], $C+(K \cap H) /(K \cap H)$ is Cartan in $K /(K \cap H)$. By the first isomorphism theorem, $C+H / H$ is Cartan in $K+H / H$. By Engel's theorem [8], $C$ acts nilpotently on $A / H$ since $A=H+E_{A}(c)$ for all $c$, hence also on $(K+H) / H$. Then there exist a flag of $(C+H) / H$-modules in $(K+H) / H$ on which $(C+H) / H$ acts nilpotently. Hence, if $(C+H) / H$ is properly contained in $(K+H) / H$, then there is an element in $(C+H) / H$ which is in the normalizer of $(C+H) / H$. This contradicts that $(C+H) / H$ is a Cartan sublagebra of $(K+H) / H$. Hence $(C+H) / H=(K+H) / H$. Therefore $(K+H) / H$ is nilpotent, as is $K+H$ since $H$ is generalized Frattini in $A$. Hence $K$ is nilpotent and $K=C$. Since $c \in C=K$ and $K$ is an ideal, $A=E_{A}(c)$.

Conversely, suppose that $H$ satisfies the conditions in the theorem. Let $K$ be an ideal with $H \subseteq K$ with $K / H$ nilpotent. Let $C$ be a Cartan subalgebra of $K$ and let $c \in C$ be an element such that $E_{A}(c)$ is minimal in the set of Engel subalgebras for $c \in C$. From Theorem 6.5 in [2], $C=E_{K}(c)$. Since $K / H$ was chosen to be nilpotent, $K=C+H=E_{K}(c)+H$. Thus $A=E_{A}(c)+H$ since $K$ is an ideal in $A$. Hence $A=E_{A}(c)$ by hypothesis. Therefore, $K=E_{K}(c)=C$ which is nilpotent. Hence $H$ is generalized Frattini by Theorem 2.7.

\section{Primitive ideals}

Definition 3.1. An ideal $K$ of $A$ is primitive if

(1) $\Phi(A / K)=0$,

(2) $A / K$ contains a unique minimal ideal,

(3) and $\operatorname{dim}(A / K)>1$.

Example 3.2. Let $A$ be the three dimensional cyclic Leibniz algebra generated by a with $a a^{3}=a^{2}$. Let $K$ be the ideal with basis $a^{2}+a^{3}$. Then $A / K$ has basis a, $a^{2}$ where we delete $K$ from the notation. $A / K$ is cyclic with generator a and $a a^{2}=-a^{2}$. The minimum polynomial for $L_{a}$ is $x(x+1)$. Thus $A / K$ has 2 maximal subalgeras and $\Phi(A / K)=0$ using section 4 of [5] and has a unique minimal ideal. Hence $K$ is a primitive ideal in $A$.

Lemma 3.3. Let $K$ be a primitive ideal in $A$. Then $K$ contains $\Phi(A)$ and $A / K$ is non-nilpotent. Hence $A$ is non-nilpotent.

Proof. Since $\Phi(\mathrm{A} / \mathrm{K})=0, \Phi(\mathrm{A}) \subseteq \mathrm{K}$. Suppose that $A / K$ is nilpotent and let $B / K$ be the unique minimal ideal of $A / K$. Now $A / K=N i l(A / K)=B / K$ by Theorem 2.4 of [4] and $\operatorname{dim}(A / K)=1$, a contradiction. Thus $A / K$ is not nilpotent. 
Proposition 3.4. Let $A$ be a solvable Leibniz algebra and let $K$ be a primitive ideal in $A$. Then $K$ is generalized Frattini in $A$ if and only if $K$ is a proper subalgebra of $\operatorname{Nil}(A)$.

Proof. Suppose that $K$ is generalized Frattini in $A$ and let $B / K$ be the unique minimal ideal of $A / K$. Since $A$ is solvable, $B / K=N i l(A / K)$ using Theorem 2.4 of [4]. Then $B / K=N i l(A / K)=\operatorname{Nil}(A) / K$ by Proposition 2.13. Hence $N i l(A)=B$ and $K$ is a proper subalgebra of $N i l(A)$.

Conversely, let $K$ be a primitive ideal in the solvable Leibniz algebra $A$ and $K$ be properly contained in $\operatorname{Nil}(A)$. Then $A / K$ contains a unique minimal ideal, $B / K$, and $\operatorname{Nil}(A) / K$ is an ideal. Therefore $B$ is contained in $\operatorname{Nil}(A)$. Since $\phi(A / K)=$ $0, B / K$ is complemented by a maximal subalgebra $M / K$ in $L / K$. Furthermore $\operatorname{Nil}(A / K)=$ the sum of all minimal ideals which is $B / K$. By assumption, $K$ is properly contained in $\operatorname{Nil}(A)$, hence $B$ is contained in $N i l(A)$. Since $B / K=$ $\operatorname{Nil}(A / K), B=\operatorname{Nil}(A)$. Let $H$ be an ideal in $A$ and let $K$ be contained in $H$. If $H / K$ is nilpotent, then $H$ is contained in $B$ and $H$ is nilpotent. Hence $K$ is generalized Frattini in $A$.

Theorem 3.5. Let $A$ be a solvable Leibniz algebra and let $K$ be a primitive ideal in $A$. Let $B / K$ be the unique minimal ideal in $A / K$. Then $K$ is generalized Frattini in $A$ if and only if $B=\operatorname{Nil}(A)$.

Proof. Suppose that $K$ is generalized Frattini in $A$. Then $N i l(A / K)=N i l(A) / K$ by Theorem 2.7. Since $A$ is solvable, $B / K=N i l(A / K)=N i l(A) / K$ and $B=$ $\operatorname{Nil}(A)$.

Conversely, suppose that $B=\operatorname{Nil}(A)$. Since $A$ is solvable, $\operatorname{Nil}(A / K)=B / K=$ $N i l(A) / K$ and $K$ is a proper subalgebra of $N i l(A)$. If $N$ is any ideal of $A$ with $N / K$ nilpotent, then $N$ is nilpotent. Hence $K$ is generalized Frattini in $A$ by Theorem 2.7 .

Corollary 3.6. Let $K$ be a primitive ideal of a solvable Leibniz algebra A. If $K$ is generalized Frattini in $A$, then $K$ is maximal with respect to the generalized Frattini property in A.

Proof. Suppose that $H$ is generalized Frattini ideal in $A$ such that $K \subseteq H$. Then $H$ is nilpotent by Proposition 2.1 and, hence, $H$ is contained in $\operatorname{Nil}(A)$. Let $B / K$ be the unique minimal ideal in $A / K$. By Theorem $3.5, B=N i l(A)$. Hence, either $H=K$ or $H=\operatorname{Nil}(A)$ since $K \subseteq H \subseteq \operatorname{Nil}(A)$. Suppose that $H=\operatorname{Nil}(A)$. Then, by Proposition 2.10, every solvable ideal of $A$ is nilpotent. In particular, $A$ 
is nilpotent. This contradicts Lemma 3.3. Thus $H=K$ and $K$ is maximal with respect to the generalized Frattini property.

\section{Intersections of certain maximal subalgebras}

Let $R(A)$ be the intersection of all maximal subalgebras that are ideals in $A$ and $T(A)$ be the intersection of all maximal subalgebras that are not ideals in $A$. As for $F(A), T(A)$ may not be an ideal and we let $\tau(A)$ be the largest ideal of $A$ contained in $T(A)$. Of course $\Phi(A)=R(A) \cap \tau(A)$.

Definition 4.1. An algebra is power solvable if all subalgebras generated by a single element are solvable.

For Leibniz algebras, these subalgebras are cyclic subalgebras in which $A^{2}=$ $\operatorname{Leib}(A)$ is abelian (Section 4 of [5]). Hence Leibniz algebras are power solvable and the following result, Theorem 2.8 of [15] holds.

Lemma 4.2. If $\Phi(A)=0$, then $\tau(A)=Z(A)=Z^{*}(A)$ where $Z^{*}(A)$ is the final term in the upper central series of $A$.

Proposition 4.3. $\tau(A)$ is generalized Frattini in $A$.

Proof. By the last lemma, $\tau(A) / \Phi(A)=Z(A) / \Phi(A)=Z(A / \Phi(A))$. By Proposition 2.1, $Z(A / \Phi(A))$ is generalized Frattini in $A / \Phi(A)$. Thus $\tau(A)$ is generalized Frattini in $A$.

Proposition 4.4. Let $A$ be a non-nilpotent Leibniz algebra with $\Phi(A)=0$. Then any ideal $H$ that is a maximal generalized Frattini ideal in $A$ contains $\tau(A)$.

Proof. By Lemma $4.2 H+Z(A)=H+\tau(A)$ which is generalized Frattini by Proposition 2.1. Using maximality of $H, H=H+\tau(A)$. Hence $\tau(A) \subseteq H$.

Theorem 4.5. $A$ is nilpotent if and only if $R(A) \subseteq \tau(A)$.

Proof. If $A$ is nilpotent, then all maximal subalgebras are ideals in $A$ Theorem 4.16 of [10]. Hence $\tau(A)=A$ and $R(A) \subseteq \tau(A)$. Conversely, if $R(A) \subseteq \tau(A)$, then $\Phi(A)=R(A)$ which contains $A^{2}$ by Lemma 2.3 of [15]. Hence all maximal subalgebras are ideals and $A$ is nilpotent.

Corollary 4.6. $A$ is nilpotent if and only if $\Phi(A)=A^{2}$.

Proof. If $A$ is nilpotent, then all maximal subalgebras are ideals by Theorem 4.16 of [10]. Then $A^{2}$ is contained in every maximal subalgebra. Hence $A^{2}$ is contained 
in $F(A)=\Phi(A)$. The reverse inclusion always holds. Conversely, if the condition holds, then all maximal subalgbras are ideals and $A$ is nilpotent by Theorem 4.16 of $[10]$.

Lemma 4.7. Let $N$ be an ideal in $A$.

(1) $F(A)+N / N \subseteq F(A / N)$.

(2) If $N \subseteq F(A)$, then $F(A) / N=F(A / N)$.

Proof. (1) If $M / N$ is a maximal subalgebra of $A / N$, then $M$ is a maximal sub-

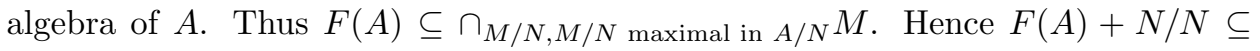
$F(A / N)$.

(2) $N$ is contained in all maximal subalgebras of $A . M / N$ is a maximal subalgebra of $A / N$ if and only if $M$ is a maximal subalgebra of $A$.Then $F(A) / N=F(A / N)$.

Let $n \operatorname{Frat}(A)$ be the intersection of all maximal ideals of $A$. Then following the same arguments as in the last lemma, we obtain:

Lemma 4.8. Let $N$ be an ideal in $A$.

(1) $n \operatorname{Frat}(A)+N / N \subseteq n \operatorname{Frat}(A / N)$.

(2) If $N \subseteq n$ Frat $(A)$, then $n \operatorname{Frat}(A) / N=n \operatorname{Frat}(A / N)$.

In Lie algebras, the Frattini ideal is nilpotent. However, $n$ Frat $(A)$ and $R(A)$ need not be nilpotent. The same results hold for Leibniz algebras also. For $x$ in $A, L_{x}$ denotes left multiplication by $x$ and $A_{0}(x)$ and $A_{1}(x)$ are the corresponding Fitting components for $L_{x} . A_{0}(x)$ is the Engel subalgebra from Section 2.

Proposition 4.9. $\Phi(A)$ is nilpotent, but $n F r a t(A)$ and $R(A)$ need not be nilpotent.

Proof. Using Proposition 2.1, $\Phi(A)$ is nilpotent. For the examples, let $A=g l(p, F)$ where $F$ has characteristic $p$. The $Z(A) \subset A^{2}=s l(p, F)$ which is the only maximal ideal of $A$ and $n \operatorname{Frat}(A)$ is not nilpotent. Since $\operatorname{sl}(p, F)$ is the only maximal subalgebra that is an ideal, $R(A)=s l(p, F)$ is not nilpotent.

Both $\Phi(A)$ and $n F r a t(A)$ are contained in $R(A)$. We find other results of this type.

Proposition 4.10. $\Phi(A) \subseteq n F r a t(A) \subseteq R(A)$.

Proof. If $N$ is a maximal ideal of $A$, then $\Phi(A)+N$ can not equal $A$, hence $\Phi(A) \subseteq N$. Hence $\Phi(A) \subseteq n F r a t(A)$. The other inclusion is clear.

Proposition 4.11. If $A$ is solvable, then $R(A)=n$ Frat $(A)$. 
Proof. Let $N$ be a maximal ideal of a solvable Leibniz algebra $A$. Then $(\mathrm{A} / \mathrm{N})^{2}=0$ and $\mathrm{A}^{2}$ is contained in $\mathrm{N}$. Hence $\operatorname{dim}(\mathrm{A} / \mathrm{N})=1$. Thus every maximal ideal is a maximal subalgebra that is an ideal. The converse also holds. Hence the result holds.

If $A$ is not solvable, then Proposition 4.11 does not hold.

Example 4.12. Let $A=\operatorname{sl}(2, F)$. Since $A$ is simple, $n$ Frat $(A)=0$. Since $A$ contains no maximal subalgebras that are ideals, $A=R(A)$.

Theorem 4.13. $A$ is nilpotent if and only if $\Phi(A)=n$ Frat $(A)=R(A)$.

Proof. If $A$ is nilpotent, then all maximal subalgebras are ideals and $R(A)=\Phi(A)$ and Proposition 4.10 gives the result.

Now suppose that the three ideals are equal. Since $\tau(A)$ always contains $\Phi(A)$, $R(A) \subseteq \tau(A)$. Then $A$ is nilpotent by Theorem 4.5 .

\section{Generators}

In groups and Lie algebras, the Frattini subgroup and Frattini subalgebra, are the sets of non-generators in the group and Lie algebra respectively. It has also been shown these concepts carry over to $R(G)$ and $n F r a t(G)$ in a group $G$ and also to $R(L)$ and $n$ Frat $(L)$ in a Lie algebra $L$ [13]. Following the results in groups and Lie algebras, we give Leibniz algebra characterizations of $F(A), R(A)$ and $n$ Frat $(A)$ in terms of non-generators.

Definition 5.1. An element $x \in A$ is a non-generator in $A$ if whenever $A=\langle x, S\rangle$ it follows that $A=\langle S\rangle$ for each subset $S$ of $A$.

Definition 5.2. A subset $S$ of $A$ that is closed under multiplications by elements of $A$ is called a normal set in $A$.

Definition 5.3. An element $x \in A$ is a normal non-generator in $A$ if whenever $A=\langle x, T\rangle$ it follows that $A=\langle T\rangle$ for each normal subset $T$ of $A$.

Proposition 5.4. $F(A)$ consists of the non-generators of $A$.

Proof. Let $x \in F(A)$ and let $A=\langle x, H\rangle$ where $H$ is a subalgebra of $A$. If $H \neq A$, then $H \subseteq M$ for some maximal subalgebra of $A$. Then $\langle H, x\rangle \subseteq M$, a contradiction. Hence $A=H$ and $x$ is a non-generator of $A$. Suppose that $x$ is not in $F(A)$. Let $M$ be a maximal subalgebra of $A$ which does not contain $x$. Then $M \subset\langle x, M\rangle=A$. But $M \neq A$, so $x$ is a not a non-generator of $A$. 
Proposition 5.5. $R(A)$ equals the set of normal non-generators of $A$.

Proof. Suppose that $x \in R(A)$ and $A=\langle x, S\rangle$ where $S$ is a normal subset of $A$. Suppose that $A \neq\langle S\rangle$. Then $B=A /\langle S\rangle$ is generated by one element, the image of $x$ in $B$. Hence $B$ is cyclic and, therefore, solvable. $B$ is generated by the image of $x$ in $B$. The derived algebra, $B^{2}$ of $B$ has codimension 1 in $B$ and is an ideal in $B$. Hence, the preimage, $C$, of $B^{2}$ in $A$ is a maximal subalgebra of $A$ that is an ideal in $A$. Hence, by assumption $x \in C$ and the image of $x$ in $B$ is in $B^{2}$. This contradicts that the image of $x$ in $B$ generates $B$. Therefore, $A=\langle S\rangle$ and $x$ is a normal non-generator of $A$

Conversely, suppose that $x \notin R(A)$. Then $x$ is not in a maximal subalgebra, $M$, that is an ideal in $A$. $M$ is a normal subset of $A$ and $A=\langle x, M\rangle$ but $A \neq M$. Hence $x$ is not a normal non-generator in $A$.

Definition 5.6. Let $X$ be a subset of $A$. Let $X^{A}$ be the smallest ideal in $A$ that contains $X$. An element $x \in A$ is called an $n$-nongenerator if for every subset $X$ of $A, A=X^{A}$ whenever $A=\langle x, X\rangle^{A}$.

Lemma 5.7. For any $x \in A$ and any subset $X$ of $A,\langle x, X\rangle^{A}=\left\langle x^{A}, X^{A}\right\rangle=$ $x^{A}+X^{A}$.

Proof. Both $\mathrm{x}^{A}$ and $\mathrm{X}^{A}$ are contained in $\langle x, X\rangle^{A}$. Therefore $\left\langle x^{A}, X^{A}\right\rangle \subseteq<$ $x, X>^{A}$ and $x^{A}+X^{A} \subseteq<x, X>^{A}$. Since $<x, X>\subseteq<x^{A}, X^{A}>$, it follows that $\left\langle x, X>^{A} \subseteq<x^{A}, X^{A}>\right.$. Also $x^{A}, X^{A} \subseteq x^{A}+X^{A}$. Thus $\left\langle x^{A}, X^{A}>\subseteq\right.$ $x^{A}+X^{A}$.

Proposition 5.8. $n$ Frat $(A)$ is the set of n-nongenerators for $A$.

Proof. Let $T$ be the collection of all $n$-nongenerators of $A$. Suppose $x$ is in $T$ but not in $n \operatorname{Frat}(A)$. Let $N$ be a maximal ideal such that $x$ is not in $N$. Then $x^{A}+N=A$. Hence $\langle x, N\rangle^{A}=A$. Thus $N=N^{A}=A$, a contradiction. Hence $T \subseteq n \operatorname{Frat}(A)$.

Conversely, let $x$ is in $n$ Frat $(A)$ but not in $T$. There exists a subset $S$ of $A$ such that $A=\langle x, S\rangle^{A}$ but $S^{A}$ is properly contained in $A$. Therefore $S^{A}$ is a proper ideal of $A$ and $x$ is not in $S^{A}$. By Lemma 5.7, $A=\langle x, S\rangle^{A}=x^{A}+S^{A}$. Let $M$ be maximal with respect to the properties for $S^{A}: x$ is not in $M, M$ is an ideal of $A, S^{A} \subseteq M$ and $A=x^{A}+M$. We claim that $M$ is a maximal ideal of $A$. If not, let $N$ be an ideal properly between $M$ and $A$. Then $A=x^{A}+M=x^{A}+N$. By the maximality conditions on $M, x \in N$. Therefore, $x^{A} \subseteq N$ and $A=N$, a contradiction. Thus $M$ is a maximal ideal in $A$. Since $x$ is not in $M$, it follows that 
$x$ is not in $n \operatorname{Frat}(A)$, a contradiction. Hence, whenever $A=\langle x, S\rangle^{A}$, it follows that $A=S^{A}$ and $x$ is an $n$-nongenerator of $A$. Hence $n \operatorname{Frat}(A) \subseteq T$ and the result holds.

Acknowledgement. The authors would like to thank the referee for their valuable comments and suggestions to improve the quality of the paper.

\section{References}

[1] Sh. A. Ayupov and B. A. Omirov, On Leibniz algebras, Algebra and operator theory (Tashkent, 1997), Kluwer Acad. Publ., Dordrecht, (1998), 1-12.

[2] D. W. Barnes, Some theorems on Leibniz algebras, Comm. Algebra, 39(7) (2011), 2463-2472.

[3] D. W. Barnes, Schunck classes of soluble Leibniz algebras, Comm. Algebra, 41(11) (2013), 4046-4065.

[4] C. Batten, L. Bosko-Dunbar, A. Hedges, J. T. Hird, K. Stagg and E. Stitzinger, A Frattini Theory for Leibniz algebras, Comm. Algebra, 41(4) (2013), 15471557.

[5] C. Batten Ray, A. Combs, N. Gin, A. Hedges, J. T. Hird and L. Zack, Nilpotent Lie and Leibniz algebras, Comm. Algebra, 42(6) (2014), 2404-2410.

[6] C. Batten Ray, A. Hedges and E. Stitzinger, Classifying several classes of Leibniz algebras, Algebr. Represent. Theory, 17(2) (2014), 703-712.

[7] J. C. Beidleman and T. K. Seo, Generalized Frattini subgroups of finite groups, Pacific J. Math., 23 (1967), 441-450.

[8] L. Bosko, A. Hedges, J. T. Hird, N. Schwartz and K. Stagg, Jacobson's refinement of Engel's theorem for Leibniz algebras, Involve, 4(3) (2011), 293-296.

[9] T. Burch, M. Harris, A. McAlister, E. Rogers, E. Stitzinger and S. M. Sullivan, 2-recognizeable classes of Leibniz algebras, J. Algebra, 423 (2015), 506-513.

[10] I. Demir, K. Misra and E. Stitzinger, On some structures of Leibniz algebras, Recent advances in representation theory, quantum groups, algebraic geometry, and related topics, Amer. Math. Soc., Providence, RI, Contemp. Math., 623 (2014), 41-54.

[11] L.-C. Kappe and J. Kirkland, Some analogues of the Frattini subgroup, Algebra Colloq., 4(4) (1997), 419-426.

[12] J.-L. Loday, Une version non commutative des algebres de Lie: les algebres de Leibniz, Enseign. Math., 39 (1993), 269-293.

[13] K. Stagg, Analogues of the Frattini subalgebra, Int. Electron. J. Algebra, 9 (2011), 124-132. 
[14] D. A. Towers, A Frattini theory for algebras, Proc. London Math. Soc., 27 (1973), 440-462.

[15] D. Towers, Two ideals of an algebra closely related to its Frattini ideal, Arch. Math. (Basel), 35(1-2) (1980), 112-120.

\author{
Allison McAlister and Ernie Stitzinger \\ Department of Mathematics \\ North Carolina State University \\ Raleigh, North Carolina, United States of America \\ e-mails: armcalis@ncsu.edu (A. McAlister) \\ stitz@ncsu.edu (E. Stitzinger)
}

Kristen Stagg Rovira (Corresponding Author)

Department of Mathematics

California State University, Dominguez Hills

Carson, California, United States of America

e-mail: kstagg@csudh.edu 